\title{
Improvement in attention following surgery treatment in pituitary adenoma patients: an evidence from ERP study
}

Chenglong Cao

southern medical university

Jian Song

southern medical university

Yujing Huang

University of Cambridge

Binbin Liu

wuhan medical college

Yu Wang

south central university for nationalities

Guozheng Xu ( $\nabla$ docxgz@163.com )

Southern Medical University https://orcid.org/0000-0001-8132-1954

Research article

Keywords: Pituitary adenoma, event-related potentials, attention, improvement, P200

Posted Date: January 3rd, 2020

DOI: https://doi.org/10.21203/rs.2.19964/v1

License: (c) (i) This work is licensed under a Creative Commons Attribution 4.0 International License.

Read Full License 


\section{Abstract}

Background: Cognitive abilities are impaired in patients with pituitary adenoma, which are mostly treated with surgery. However, the study on neurocognitive recovery after transsphenoidal adenomectomy is lacking. The study aims to identify the electrophysiological change that relates to the attention function of pituitary adenoma patients after treatment. Methods: 27 preoperative pituitary patients and 25 follow up postoperative patients were recruited. 27 healthy controls ( $\mathrm{HCs}$ ) were matched to the patients with age, sex, and education. All the pituitary adenoma patients had successfully gone through transsphenoidal adenomectomy under microscope or endoscope. Event-related potentials were used to investigate the cognitive processes of attention for preoperative patients, postoperative patients and healthy controls. In addition, eyesight and blood hormone levels were examined. Six months after surgery, blood hormone levels, pituitary MRI and electrophysiological tests were re-examined. Results: Across three groups, all emotional stimuli could evoke P200 components. Compared with HCs or postoperative patients, the amplitudes of P200 in the preoperative patients were higher. Moreover, The amplitude of P200 decreased in postoperative patients, which was similar to that in HCs. The attention was improved after surgery, but no significant differences were detected between postoperative patients and HCs. Conclusion: The tumor mass effect and abnormal hormone may be relevant to the factors that impair the attention. Compared with that of the HCs and postoperative patients, the P200 component elicited by negative stimuli is higher in preoperative patients, which is associated with the attention impairments. Furthermore, these data indicate the improvement in attention may be attributed to the tumor resection and the amelioration of endocrine disorders. This study shows that P200 component can be used to diagnose the attention in preoperative pituitary patients, and prove the improvement of the attention in postoperative patients.

\section{Background}

Pituitary adenomas are the most common intracranial tumors following meningiomas, accounting for about $16.5 \%$ of central nervous system tumors [1], and pituitary adenoma with unconspicuous symptoms has a higher incidence. The mechanical pressure from tumor mass on adjacent neuroanatomical regions such as inferior frontal lobe, diencephalon, optic chiasma, and pituitary stalk could disrupt the tissue structures [2]-[6], which might decrease the endocrine functions of hypothalamus or pituitary stalk. On the other hand, the pituitary may secrete abnormally high hormones than usual. Therefore, apart from the physical damages, abnormal cognition appears commonly in pituitary adenoma patients. Patients frequently complain about problems of memory and sustained attention, which affect the quality of patients' life. The cognitive deficits of pituitary patients often include dysfunction in attention processes [6][7]. Moreover, many studies on affective neuroscience have identified neural networks distributed in the brain and involved in the processing of emotional expressions on face. These neural networks include the prefrontal, basal ganglia and amygdala regions, which contribute to emotional detection and semantic processing [8]. Our team has found that attention and executive function are influenced by pituitary adenoma [9][10]. 
There are various neuropsychological and behavioral tests to assess the cognitive function [11][12]. Since the relationship between the event-related potentials (ERPs) and cognitive performance was identified, many researchers began to study the clinical significance of ERPs in order to understand electrophysiological mechanisms of cognition [13]. Up to date, no electrophysiological studies of attention have been conducted on pre and postoperative pituitary patients, which will be discussed in the present study by recording and analyzing P200 elicited by emotional stimuli. P200, a part of early positive ERPs component, has a peak latency from 100 to $200 \mathrm{~ms}$. P200 is an indicator of the attention bias occurring automatically [14], and also sensitive to the emotional stimuli [15]. Previous study has shown that P200 is produced immediately after detection of threatening stimuli, such as frightful images [16]. We hypothesized that preoperative patients would show abnormal attention function. Moreover, postoperative patients would show improved performance on attention processing and perform equally to the healthy controls (HCs), due to the tumor resection and the amelioration of endocrine disorders.

\section{Methods}

\section{Subject selection}

Pituitary patients with prolactinoma were recruited in the Department of Neurosurgery, Central Theater Command General Hospital of the Chinese People's Liberation Army after definite diagnosis. From October 2017 to April 2018, 27 pituitary patients took part in our study. These participants were diagnosed as pituitary adenoma by brain MRI before surgery, then by pathological detection after surgery, and met all the following inclusion criteria: (1) tumor resection was based on magnetic resonance imaging (MRI) brain scan and no compression to adjacent tissues was left after instant and 6 months surgery; (2) blood hormone levels were controlled in normal range; (3) no history of craniotomy and radiation therapy; (4) participants could cooperate to complete ERPs tests. Patients were excluded if they met one of the following conditions: (1) they had a history of neurologic or psychiatric disorders; (2) they had other disease that can affect cognitive functions like severe liver, heart or kidney dysfunction; (3) the surgery was not successful or the patients had severe complications, such as coma, infection, epilepsy, hydrocephalus and leaking of cerebrospinal fluid; (4) they had drug or alcohol abuse [subjects who drink alcohol over 2.0 standard drinks ( $10 \mathrm{~g}$ of pure alcohol) on days and meet any 2 of the 11 criteria under the DSM-V (Diagnostic and Statistical Manual of Mental Disorders) in the past year] [17], medication intake (including oral contraceptives). MRI demonstrated that the anatomical conditions were normal without any suprasellar mass or sellar- or para-sellar tissue displacement. 25 follow up patients after 6 months were included into the postoperative group.

All patients and HCs had sufficient visual acuity and hearing ability for the study. There were 27 preoperative patients (female, 15; $27.9 \pm 2.8 \mathrm{y}$ ) and 25 postoperative patients (female, 13; $27.6 \pm 2.2 \mathrm{y}$ ) with prolactin adenoma, growth hormone adenoma or non-functional pituitary adenoma. 27 healthy controls (female, $14 ; 27.2 \pm 2.9 \mathrm{y}$ ) were selected into the HCs. Healthy people did not have any mental disorders and family history of mental disease as well as psychoactive substance abuse. There was no statistic difference in age, gender, educational level among the 27 preoperative, 25 postoperative patients 
and $27 \mathrm{HCs}(p>0.05)$. The study was approved by the ethic committee of the Central Theater Command General Hospital of the Chinese People's Liberation Army and informed consents were understood and signed by the patients themselves.

\section{Endocrinological assessment}

After $12 \mathrm{~h}$ fasting, vein blood was collected between 8:00 a.m. and 9:30 a.m. due to circadian change of hormone. Then the clotted and heparinized blood samples were put onto ice in the super clean bench of the clinical laboratory of the hospital. Serum levels of prolactin $(P R L)(n g / m l)$, follicle stimulating hormone $(\mathrm{mlU} / \mathrm{ml})$, estradiol $\mathrm{ag} / \mathrm{ml})$, luteinizing hormone $(\mathrm{mlU} / \mathrm{ml})$, testosterone $(\mathrm{ng} / \mathrm{ml})$, progesterone $(\mathrm{ng} / \mathrm{ml})$, thyroid-stimulating hormone (ulU/ml), growth hormone $(\mathrm{ng} / \mathrm{ml})$, and cortisol $(\mathrm{nmol} / \mathrm{l})$ were detected by immunometric assay (Roche, cobas ${ }^{\circledR} 8000$, Switzerland) [18]. After an hour of blood collection, the cognitive function was evaluated. Before surgery, there were 21 patients with prolactinomas, 4 patients with growth hormone adenomas and 2 patients with non-functioning cases. 25 follow up patients were included into the postoperative group, including 20 patients with prolactinomas, 3 patients with growth hormone and 2 patients with non-functional pituitary adenoma.

\section{Stimuli and procedure}

Affective stimulis were presented in grey background in the central monitor (light degree is set to $60 \mathrm{~cd} / \mathrm{m} 2$ ). Participants watched the screen $100 \mathrm{~cm}$ away from their eyes in a semi-dark room, with a visual angle of $4^{\circ} \times 4^{\circ}$. Forty-five pictures (15 negative, 15 neutral, 15positive) were selected from IAPS (International Affective Picture System) images [19]. Table. 1 represents the means and standard deviations of both dimensions (arousal and valence) for each type of stimulus. One-way analysis of variance (ANOVA) were computed for valence and for arousal dimensions. The positive and negative pictures were matched for perceived arousal. They did not differ significantly from each other but differed significantly from the neutral ones (P凶0.05). As for valence, positive pictures were significantly higher than neutral ones (Pख0.01) and neutral pictures were significantly higher than negative ones (Pख0.01). On each trial, there was only one image (positive, negative, or neutral image) presented on the center of the screen. The trial began with a fixation mark (+) on the black screen for $1000 \mathrm{~ms}$, and then an image appeared for $2000 \mathrm{~ms}$. Ten pictures, different from the experimental trials, served as practice trials. The inter-trial interval varied between $2000 \mathrm{~ms}$ and $3000 \mathrm{~ms}$. Participants were instructed to attentively view each picture and to mentally categorize it either as a positive, negative or neutral picture after stimulus offset. Pictures were presented in a randomized order in three blocks, with 45 pictures per block, with no more than two pictures of the same stimulus condition being shown in succession. We just instruct participants to mentally categorize these stimuli and without any pressing button, only in order to pay their attention on these stimuli. The images were presented in a pseudo random order, with the constraint that a specific image appeared no more than two times consecutively. During the trial, the subjects were instructed to look passively at the pictures, and reduce eye blinks and body movements as much as they can.

\section{Electroencephalography (EEG) recording and analysis}


The EEG was acquired by a 64-channel array (eegoTM amolifier, Germany) linked to both earlobe reference electrodes built in an elastic cap. The impedance levels of EEG recording were under $5 \mathrm{~K} \Omega$. EEG signals were continuously recorded with a band pass of $0.05-200 \mathrm{~Hz}$. The sampling rate was at $1000 \mathrm{HZ}$ during acquisition, then was resampled at $250 \mathrm{HZ}$. Based on previous reports [20][21], we performed ANOVAs with anterior-posterior (AP) distribution (three levels: anterior, central, posterior sites) and lateral distribution (three levels: left, midline, right sites) as within-subject factors. The EEG data were off-line referenced to average reference of all electrodes. The EEG segment was between $100 \mathrm{~ms}$ pre-stimulus [22][23] and 1000 ms post-stimulus, and the baseline was corrected to the mean amplitude $100 \mathrm{~ms}$ prestimulus. The negative, neutral and positive epoches were averaged respectively. At least 40 trials were made for each subject. The filter frequency is $1 \mathrm{HZ}$ high-pass, $40 \mathrm{HZ}$ low-pass. We used repeatedmeasures analysis of variance (ANOVA) to acquire effects of picture type and analyze whether these effects are different over the electrode sites of the mean amplitudes of P200 and among different groups. The degrees of freedom in our study were corrected with Greenhouse-Geisser

\section{Result}

Figure 1 shows the mean ERPs of HCs at the nine ERP electrodes within the time window of P200 (148 ms-252 ms) evoked by the three kinds of stimulus respectively. Obviously, compared with the neutral stimuli in the same control group, negative stimuli induced decreased negative potentials in P200 time window. Figure 2 shows the mean ERPs of the three groups' response to negative stimuli at the $9 \mathrm{ERP}$ locations. Figure 3 shows the average ERPs evoked by emotional stimuli and neutral stimuli in the middleline areas $(\mathrm{Fz}, \mathrm{Cz}, \mathrm{Pz})$ in the preoperative group, postoperative group and the control group respectively.

With respect to P200 amplitudes, there were no remarkable different effects, though we obtained significant interactions of Stimulus type ${ }^{\star} \operatorname{Group}(p=0.01)$, but no interaction effect of Hemisphere ${ }^{\star}$ Group $(p=0.17)$. Post-hoc results showed that the effect of the Stimuli was clearly significant in the control group $(p=0.001)$. The negative stimuli $(-0.17 \mu \mathrm{V})$ evoked decreased amplitudes of P200 compared with the positive and neutral stimuli in the control group (neutral $=0.42 \mu \mathrm{V}$, positive $=0.21 \mu \mathrm{V}$ )(Fig. 1). In response to negative stimuli of the three groups, post-hoc analyses indicated that differences between both preoperative $(0.53 \mu \mathrm{V})$ and postoperative $(-0.09 \mu \mathrm{V}, \mathrm{p}=0.006)$ and preoperative $(0.53 \mu \mathrm{V})$ and healthy controls $(-0.17 \mu \mathrm{V}, p=0.002)$ were significant, without differences between postoperative

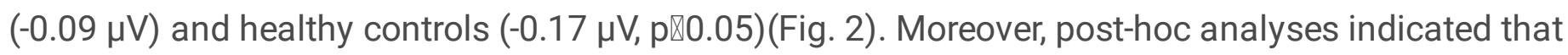
differences between both preoperative $(0.86 \mu \mathrm{V})$ and postoperative $(0.24 \mu \mathrm{V}, \mathrm{p}=0.01)$ and preoperative $(0.86 \mu \mathrm{V})$ and healthy controls $(0.24 \mu \mathrm{V}, p=0.02)$ were significant at middleline areas, but no difference

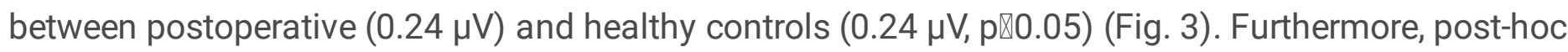
analyses revealed decreased amplitudes in the postoperative $(0.17 \mu \mathrm{V})$, compared to the preoperative $(0.91 \mu \mathrm{V}, \mathrm{p}=0.04)$ at central electrode.

\section{Discussion}


In the present medical study of pituitary adenoma, much attention has been paid to the surgery itself for a long time. However, few studies have investigated the patient's attention function and the recovery after treatment. Our study evaluated the attention dysfunction in preoperative pituitary patients compared to HCs. Here, we also sought to assess the recovery of attention in patients after treatment.

The association of the concrete operation and its effects on cognitive functions are still inconsistent [24]. For instance, Peace et al [6] found that the transfrontal operation caused more severe injury to cognition, but Guinan et al [25] observed no association between the cognitive dysfunction and the treatment approach [25][26][6]. Therefore, all the patients did not go through the transfrontal operation and radiation treatment, but underwent transsphenoidal adenomectomy. In this study, we did not carry out neuropsychological test. Because our previous research [18] assessed attention processing using the Digit Span Forwards and Backwards Tests of the Wechsler Adult Intelligence Scale-Revised, Chinese version. We did not find any obvious differences between presurgical group and HCs, perhaps because of the simplicity of the present behavioral task. However, we found significant group differences for electrophysiological data.

In this study, we found no hemispheric differences in our recordings, because the asymmetry is neither very significant in some phases of the emotional reaction nor in some stimulus conditions [27][28]. Importantly, we found, compared with the positive and neutral stimuli, the negative stimuli elicited decreased amplitudes of P200 in the HCs. Previous studies indicated that negative stimuli elicited more prominent responses than neutral or positive stimuli [29][14]. However, recent research has found that the extremely negative stimuli produced smaller amplitudes of P200 than did the moderately negative and neutral stimuli over a wide region across the scalp [30]. It has been reported that there is a cultural bias for the IAPS in Chinese subjects [31]. For pictures containing intense feeling contradicted to traditional Chinese culture, their valence scores were significantly lower than that of the original subjects [32]. Frontal P200 activation is the sign of quick detection of typical stimuli [33]. Relevant to moderately negative stimuli, extremely negative stimuli often include remarkable threatening contents (e.g. bleeding), which have been shown to attract human attention resources rapidly and automatically [34][35].

Therefore, smaller P200 amplitudes in the negative condition are likely a rapid feature detection process to threatening content [35]. In line with the discussion above, we also found the negative stimuli elicited decreased amplitudes of P200 in the HCs, compared with the positive and neutral stimuli. Thus, our finding of smaller P2 amplitudes in the negative stimuli may be likely relevant to a rapid feature detection processing that attends to threatening contents [35]. Moreover, we obtained the differences among three groups at middleline areas. In response to negative stimuli of the three groups, the finding of this research showed the amplitudes of P200 in preoperative patients were larger than that in both postoperative patients and HCs. Whether preoperative patients have difficulties attracting attention resources rapidly and automatically, when they are faced with extremely negative stimuli, such as remarkable threatening stimuli. Increased P200 components are reported in high trait-anxious individuals [36]. The boost of perceptual processing has also been indicated in other types of phobic syndromes, and such a state of hypervigilance to salient stimuli might be a feature of the cognitive functioning of phobic individuals [37] [38]. It was reported that pituitary adenoma patients showed a different mode of increased anxious 
personality compared with the general population [39]. Furthermore, pituitary adenoma patients have significantly higher degree of depression and anxiety than HCs [40]. The pituitary gland is the pivot of hypothalamic-pituitary-adrenal (HPA) axis, and its activation can trigger adrenarche. One possible explanation for this finding is that an enlarged pituitary gland might be associated with hyperactivity/reactivity of the HPA axis. Weitzner et al reported that the hypothalamic-pituitary axis could affect behavioural control function of the prefrontal cortex [41]. It may be suggested that the larger amplitudes of P200 could reflect a greater involvement of intense resources on pivotal stimuli (i.e. angry faces) in pituitary patients.Because preoperative patients may have difficulties attracting attention resources rapidly and automatically, when they are faced with extremely negative stimuli, such as remarkable threatening stimuli. We postulates that anxiety in pituitary adenoma patients decreased processing efficiency through an increased use of processing resources. Pituitary patients may have difficulties to shift their attention away from threatening stimuli just like socially anxious patients [42]. Therefore, the facilitated responses to negative stimuli in preoperative patients may be these factoers. One is the compensatory mechanism that preoperative patients have difficulties attracting attention resources rapidly, so they have to employ more neurons to complete the negative stimuli processing. Previous research found an increasement of the P200 amplitude during attentional task in depressed participants, which suggested that that neural systems were attempting to compensate for a difficulty in perceiving target and non-target stimuli [43]. The other may be due to the difficulty in shifting themselves away from the threatening stimuli. Hence, the use of the P200 component during presenting affective stimuli could be used a relevant marker to investigate attention functiion.

The cognitive dysfunction may be caused by both tumor mass compression of the related brain structures and effects of related hormonal imbalance that influence cognitive structures. However, up to now, the detailed mechanism of cognitive impairments is still unexplained [44][45][46]. Hyperpituitarism can mainly cause cognitive deficits in functioning pituitary adenoma patients [46][47][48], and the oppression of the tumor to the adjacent nerve structures result in cognitive deficits caused by nonfunctioning pituitary adenomas. Suprasellar development of the tumor may destroy directly or indirectly adjacent nerve tissue, such as the hypothalamus, diencephalon, mammillary bodies, or the inferior frontal lobes. In subjects with non-functioning adenomas, successful removal of the suprasellar tumor could improve psychomotor speed, selective attention and accuracy function, which applied to both patients with non-functioning adenomas and patients with prolactinomas or sellar lesions [49]. Previous animal experiments have shown that proper PRL level plays a significant role in preventing stress-induced decrease of hippocampal neurogenesis and protect against excitotoxicity to hippocampal neurons through PRL receptors [52][53]. Nevertheless, if PRL level is too high, it can produce negative effect on cognitive abilities [46][54][56]. The association of impairments of cognitive function with hyperprolactinemia has also been found [46][54]. These cognitive dysfunctions, especially attention function, are also related to the abnormal prefrontal cortex. Previous studies have suggested that frontal/parietal cortex and subcortical structures (e.g., thalamus) were related to attention selection and shifting [57][58]. For example, recent studies specifically showed impairments in recognizing and regulating emotion in patients with temporal or frontal tumors [59]. Our study also found that patients 
with prolactinomas showed the decrease of gray matter volume (GMV) in the whole prefrontal cortex [18], suggesting that abnormal high PRL levels may have an adverse effect on cognitive function. This effect may be mediated by dopamine [60], or regulated by high level of PRL in the right IFC [61]. In addition to the physical lesion, the endocrine disorders related to functioning pituitary adenoma might be another cause of the damages.

Six months after pituitary adenoma surgery, the amplitudes of P200 in postoperative patients were similar to the HCs. Improvement in cognitive functions after surgery was related to the normal endocrine [62]. Consistently, we found that patients after successful tumor resection showed a sharply decreased P200 amplitudes. Hence, postoperative patients performed as well as HCs in attention. Because on the one hand, the suprasellar tumor was removed and former displacement of neuro-structures at or near the sellar- region was no longer visible. Subsequently, the neural structures restored, which lead to the recovery of attention. Therefore, we assume that patients suffered from suprasellar tumor, whether they are functioning or not, can profit from the decompression of adjacent neuroanatomical structures [50] [51]. On the other hand, surgery removed the tumor, reducing secretion from functioning pituitary adenoma. With physiologically normal levels of hormone, postoperative pituitary patients manifested improvement of attention, showing the similar amplitudes of P200 as HCs did.

Several limitations should be addressed. First, although there was a significantly decreased theta coherence in preoperative group, the relatively small sample size should be increased in our future study to validate the generalization of this finding. Second, The age of the patient at the time may play a role in the hormonal disorders because older adolescents are more vulnerable to suffer cognitive impairments compared to young patients [63].

\section{Conclusion}

In summary, the present study investigated the change of attention function in pituitary adenoma patients by analyzing P200 component. P200 related to negative stimuli was larger in preoperative group than the HCs and postoperative group. These data suggest the dysfunction of attention in preoperative patients and improvement of attention function in postoperative patients. Successful removal of pituitary adenomas and the restore to normal hormone levels may be associated with the recovery of attention.

\section{Abbreviations}

$\mathrm{HC}$ : healthy controls; ERPs: event-related potentials; MRI: magnetic resonance imaging; DSM-V: Diagnostic and Statistical Manual of Mental Disorders; IAPS: International Affective Picture System; EEG: Electroencephalography; AP: anterior-posterior; ANOVA: analysis of variance; HPA: hypothalamic-pituitaryadrenal; PRL: prolactin; GMV: gray matter volume.

\section{Declarations}


Acknowledgements

Not applicable.

\section{Authors' contributions}

XGZ designed the study, and supervised the study and edited the Manuscript; CCL supervised the data collection, data analysis and drafted the manuscript; SJ supervised the study and provided specialty knowledges supports; HYJ and WY provided technical and material supports; LBB contributed to the data collection. All authors have read and approved the manuscript.

\section{Funding}

This work is supported by the funding from National Natural Science Foundation of China (ID: 81571049 and 81870863).

\section{Data Availability Statement}

The datasets for this manuscript are not publicly available at this moment because the data recording is ongoing for more subjects. Future requests to access the datasets should be directed to the corresponding author.

\section{Ethics approval and consent to participate}

The authors have declared no conflict of interest in this study. The study was approved by the Institutional Review Board of Central Theater Command General Hospital of the Chinese People's Liberation Army. The number of the approved ethical statement is "[2014] 024-1." All the participants signed written informed consent form.

\section{References}

1. Ostrom Q T, Gittleman H, Truitt G, et al. CBTRUS statistical report: primary brain and other central nervous system tumors diagnosed in the United States in 2011-2015[J]. Neuro-oncology, 2018, 20(suppl_4): iv1-iv86.

2. Yamamoto T, Sakakibara R,Uchiyama T, Liu Z, Ito T,Yamanishi T, Hattori T (2005) Lower urinary tract function in patients with pituitary adenoma compressing hypothalamus. J Neurol Neurosurg Psychiatry 76(3):390-

3. Tachibana O, Yamaguchi N, Yamashima T, Yamashita J (1990) Radiation necrosis of the optic chiasm, optic tract, hypothalamus, and upper pons after radiotherapy for pituitary adenoma, detected by gadolinium-enhanced, T1-weighted magnetic resonance imaging: case report. Neurosurgery 27(4):640-

4. Kleinschmidt-DeMasters BK,Winston KR, Rubinstein D, Samuels MH (1990) Ectopic pituitary adenoma of the third ventricle. Case report. J Neurosurg 72(1):139- 
5. Peker S, Sun I, Kurtkaya-Yapicier O, Elmaci I, Pamir MN (2005) Ectopic pituitary adenoma located at the pituitary stalk. Case report. J Neurosurg Sci 49(1):25-

6. Peace K A, Orme S M, Thompson A R, et al. Cognitive dysfunction in patients treated for pituitary tumours[J]. Journal of clinical and experimental neuropsychology, 1997, 19(1): 1-6.

7. Noad, R., Narayanan, K.R., Howlett, T. et al. (2004) Evaluation of the effect of radiotherapy for pituitary tumours on cognitive function and quality of life. Clinical Oncology, 16, 233-

8. Adolphs, R. (2002). Neural system for recognizing emotion. Current Opinion in Neurobiology, 12, 169-

9. Cao CL, Song J, Yao S, et al. The dysfunction of inhibition control in Pituitary patients: Evidence from the Go/go ERP study. Neuroreport. 2017, 28 (5): 272-278.

10. Song J, Cao CL, Yang M, Yao S, et al. The dysfunction of processing task-irrelevant emotional faces in pituitary patients: an evidence from expression- related visual MMN. Neuroreport, $2018 \mathrm{Mar}$ 7;29(4):328-333.

11. Lindeboom J, Ter Horst R, Hooyer C, Dinkgreve M, Jonker C (1993) Some psychometric properties of the CAMCOG. Psychol Med 23(1):213-

12. Cockrell JR, Folstein MF (1988) Mini-mental state examination (MMSE). Psychopharmacol Bull 24(4):689-

13. Sutton, M. Braren, J. Zubin, E.R. John, Evoked-potential correlates of stimulus uncertainty, Science 150 (1965) 1187-1188.

14. Huang $Y X$, Luo $Y$ J. Temporal course of emotional negativity bias: an ERP study[J]. Neuroscience letters, 2006, 398(1-2): 91-96.

15. Wang $X$, Huang $Y, M a ~ Q$, et al. Event-related potential P2 correlates of implicit aesthetic experience. [J]. Neuroreport, 2012, 23(14):862-6.

16. Correll J , Urland G R, Ito T A . Event-related potentials and the decision to shoot: The role of threat perception and cognitive control[J]. Journal of Experimental Social Psychology, 2006, 42(1):0-128.

17. American Psychiatric Association. Diagnostic and Statistical Manual of Mental Disorders (5th ed), DSM-V. American Psychiatric Publishing (2013). 490-571 p.

18. Yao S, Song J, Gao J, et al. cognitive Function and serum hormone levels are associated with gray Matter Volume Decline in Female Patients with Prolactinomas[J]. Frontiers in neurology, 2018, 8: 742.

19. Lang P J, Bradley M M, Cuthbert B N. International affective picture system (IAPS): affective ratings of pictures and instruction manual. University of Florida, Gainesville[R]. Tech Rep A-8, 2008.

20. Delogu F, Drenhaus H, Crocker M W . On the predictability of event boundaries in discourse: An ERP investigation[J]. Memory \& Cognition, 2017.

21. Luis Carreti'ea, Francisco Mercadoa, Manuel Tapiaa, et al. Emotion, attention, and the 'negativity bias', studied through event-related potentials[J]. International Journal of Psychophysiology Official Journal of the International Organization of Psychophysiology, 2001, 41(1):75-85. 
22. Rossignol M , Philippot $P$, Cécile Bissot, et al. Electrophysiological correlates of enhanced perceptual processes and attentional capture by emotional faces in social anxiety[J]. Brain Research, 2012, 1460(none):0-0.

23. Champagne $J$, Mendrek A, Germain M , et al. Event-related brain potentials to emotional images and gonadal steroid hormone levels in patients with schizophrenia and paired controls[J]. Frontiers in Psychology, 2014, 5.

24. Donnet A, Schmitt A, Dufour $\mathrm{H}$, et al. Neuropsychological follow-up of twenty two adult patients after surgery for craniopharyngioma. Acta Neurochir (Wien) 1999;141:1049-

25. Guinan EM, Lowy C, Stanhope N, et al. Cognitive effects of pituitary tumours and their treatments: two case studies and an investigation of 90 patients. J Neurol Neurosurg Psychiatry 1998;65:870-

26. Peace KA, Orme SM, Padayatty SJ, et al. Cognitive dysfunction in patients with pituitary tumour who have been treated with transfrontal or transsphenoidal surgery or medication. Clin Endocrinol (Oxf) 1998;49:391-

27. Davidson R J . Cerebral asymmetry, emotion, and affective style.[J]. Massachusetts Institute of Technology, 1995, 12(1):361-387.

28. Spence $S$, Shapiro $D$, Zaidel $E$. The role of the right hemisphere in the physiological and cognitive components of emotional processing[J]. Psychophysiology, 1996, 33(2):112-122.

29. Delplanque $S$, Silvert $L$, Hot $P$, et al. Event-related $P 3 a$ and $P 3 b$ in response to unpredictable emotional stimuli[J]. Biological Psychology, 2005, 68(2):0-120.

30. Chen A . Are we sensitive to valence differences in emotionally negative stimuli? Electrophysiological evidence from an ERP study[J]. Neuropsychologia, 2007, 45(12):2764-2771.

31. Yuxia H, Yuejia L . Native Assessment of International Affective Picture System[J]. Chinese Mental Health Journal, 2004, 39(5-6):131-131.

32. Nan $L X$, Xiang X A , Beijing. Native Research of International Affective Picture System:Assessment in University Students[J]. Chinese Journal of Clinical Psychology, 2009.

33. Thorpe S, Fize D , Marlot C . Speed of processing in the human visual system[J]. Nature (London), 1996, 381(6582):520-522.

34. Hansen $\mathrm{CH}$, Hansen R D. Finding the face in the crowd: an anger superiority effect[J]. Journal of personality and social psychology, 1988, 54(6): 917.

35. Li X, Li X, Luo Y J . Anxiety and attentional bias for threat: an event-related potential study[J]. NeuroReport, 2005, 16(13):1501-1505.

36. Bar-Haim Y , Lamy D , Glickman S . Attentional bias in anxiety: A behavioral and ERP study[J]. Brain \& Cognition, 2005, 59(1):0-22.

37. Kolassa I T , Kolassa S , Bergmann S , et al. Interpretive bias in social phobia: An ERP study with morphed emotional schematic faces[J]. Cognition \& Emotion, 2009, 23(1):69-95.

38. Kolassa I T, Musial F, Kolassa $S$, et al. Event-related potentials when identifying or color-naming threatening schematic stimuli in spider phobic and non-phobic individuals[J]. Bmc Psychiatry, 2006, 
$6(1): 38$

39. Sievers C, Ising M, Pfister H, et al. Personality in patients with pituitary adenomas is characterized by increased anxiety-related traits: comparison of 70 acromegalic patients with patients with nonfunctioning pituitary adenomas and age- and gender-matched controls.[J]. European Journal of Endocrinology, 2009, 160(3):367-373.

40. Ragnarsson 0 , Berglund P , Eder D N , et al. Long-Term Cognitive Impairments and Attentional Deficits in Patients with Cushing's Disease and Cortisol-Producing Adrenal Adenoma in Remission[J]. J Clin Endocrinol Metab, 2012, 97(9):1640-8.

41. Weitzner M A, Kanfer S , Boothjones M. Apathy and Pituitary Disease: It Has Nothing to Do With Depression[J]. Journal of Neuropsychiatry \& Clinical Neurosciences, 2005, 17(2):159.

42. Buckner J D , Maner J K, Schmidt N B . Difficulty Disengaging Attention from Social Threat in Social Anxiety[J]. Cognitive Therapy and Research, 2010, 34(1):99-105.

43. Kemp A H, Hopkinson P J, Hermens D F, et al. Fronto-temporal alterations within the first $200 \mathrm{~ms}$ during an attentional task distinguish major depression, non-clinical participants with depressed mood and healthy controls: A potential biomarker?[J]. Human brain mapping, 2009, 30(2): $602-614$.

44. Pereira AM, Tiemensma J, Romijn JA, Biermasz NR (2012) Cognitive impairment and psychopathology in patients with pituitary diseases. Neth J Med 70(6):255-

45. Tooze A, Hiles CL, Sheehan JP (2012) Neurocognitive changes in pituitary adenoma patients after gamma knife radiosurgery: a preliminary study. World Neurosurg 78(1-2):122-

46. Bala A, Łojek E, Marchel A. Cognitive functioning of patients with a PRL-secreting pituitary adenoma A preliminary report[J]. Neurology, 2016, 86(8): 731-734.

47. Forget $H$, Lacroix A, Somma M, et al. Cognitive decline in patients with Cushing's syndrome. J Int Neuropsychol Soc 2000;6:20-

48. Sievers C, Samann PG, Pfister H, et al. Cognitive function in acromegaly: description and brain volumetric correlates. Pituitary 2012;15:350-

49. Psaras T, Milian M, Hattermann V, et al. Executive functions recover earlier than episodic memory after microsurgical transsphenoidal resection of pituitary tumors in adult patients-a longitudinal study[J]. Journal of Clinical Neuroscience, 2011, 18(10): 1340-1345.

50. Meyers CA. Neurobehavioral functioning of adults with pituitary disease. Psychother Psychosom 1998;67:168-

51. Freedman L, McFadden L. Diencephalic amnesia secondary to craniopharyngioma (abstract). J Int Neuropsychol Soc 1995;1:170.

52. Lennartsson A-K, Jonsdottir IH. Prolactin in response to acute psychosocial stress in healthy men and women. Psychoneuroendocrinology (2011) 36:1530- doi:10.1016/j.psyneuen.2011.04.007.

53. Vergara-Castañeda E, Grattan DR, Pasantes-Morales H, Pérez-Domínguez M, Cabrera-Reyes EA, Morales T, et Prolactin mediates neuroprotection against excitotoxicity in primary cell cultures of 
hippocampal neurons via its receptor. Brain Res (2016) 1636:193-9. doi:10.1016/j.brainres. 2016.02.011.

54. Henry J F, Sherwin B B. Hormones and cognitive functioning during late pregnancy and postpartum: a longitudinal study[J]. Behavioral neuroscience, 2012, 126(1): 73.

55. Montalvo I, Gutiérrez-Zotes A, Creus M, Monseny R, Ortega L, Franch J, et Increased prolactin levels are associated with impaired processing speed in subjects with early psychosis. PLoS One (2014) 9:e89428.

56. Torner L, Tinajero E, Lajud N, Quintanar-Stéphano A, Olvera-Cortés E. Hyperprolactinemia impairs object recognition without altering spatial learning in male rats. Behav Brain Res (2013) 252:32-

57. Gitelman D R, Nobre A C, Parrish T B, et al. A large-scale distributed network for covert spatial attention: further anatomical delineation based on stringent behavioural and cognitive controls[J]. Brain, 1999, 122(6): 1093-1106.

58. Morris J S , Friston K J , Dolan R J . Neural responses to salient visual stimuli[J]. Proceedings of the Royal Society B: Biological Sciences, 1997, 264(1382):769-775.

59. Campanella, F., Shallice, T., lus, T., Fabbro, F., \& Skrap, M. (2014). Impact of brain tumour location on emotion and personality: A voxel-based lesion-symptom mapping study on mentalization processes. Brain, 137, 2532-

60. Fitzgerald P, Dinan TG. Prolactin and dopamine: what is the connection? A review article. J Psychopharmacol (2008) 22:12-

61. Cabrera-Reyes EA, Limón-Morales O, Rivero-Segura NA, Camacho-Arroyo I, Cerbón M. Prolactin function and putative expression in the brain. Endocrine (2017) 57:199-

62. Wang $X$, Tong $X$, Zou $Y$, et al. The impact on cognitive functions of patients with pituitary adenoma before and after surgery[J]. Neurological Sciences, 2017, 38(7): 1315-1321.

63. Dennis M, Spiegler BJ, Obonsawin MC, et al. Brain tumors in children and adolescents-III. Effects of radiation and hormone status on intelligence and on working, associative and serial-order memory. Neuropsychologia, 1992, 30(3): 257-275.

\section{Table}

Table1 Means and standard deviations for arousal and valence for each IAPS

Mean valence and

arousal ratings Positive

Valence

Arousal
IAPS

Neutral Negative $\mathbf{P}$
$3.164 \pm 0.449 \quad \otimes 0.01$

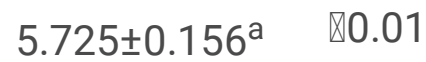


$\mathrm{a} \rrbracket P<0.05 \rrbracket$ compared with neutral stimuli;

\section{Figures}

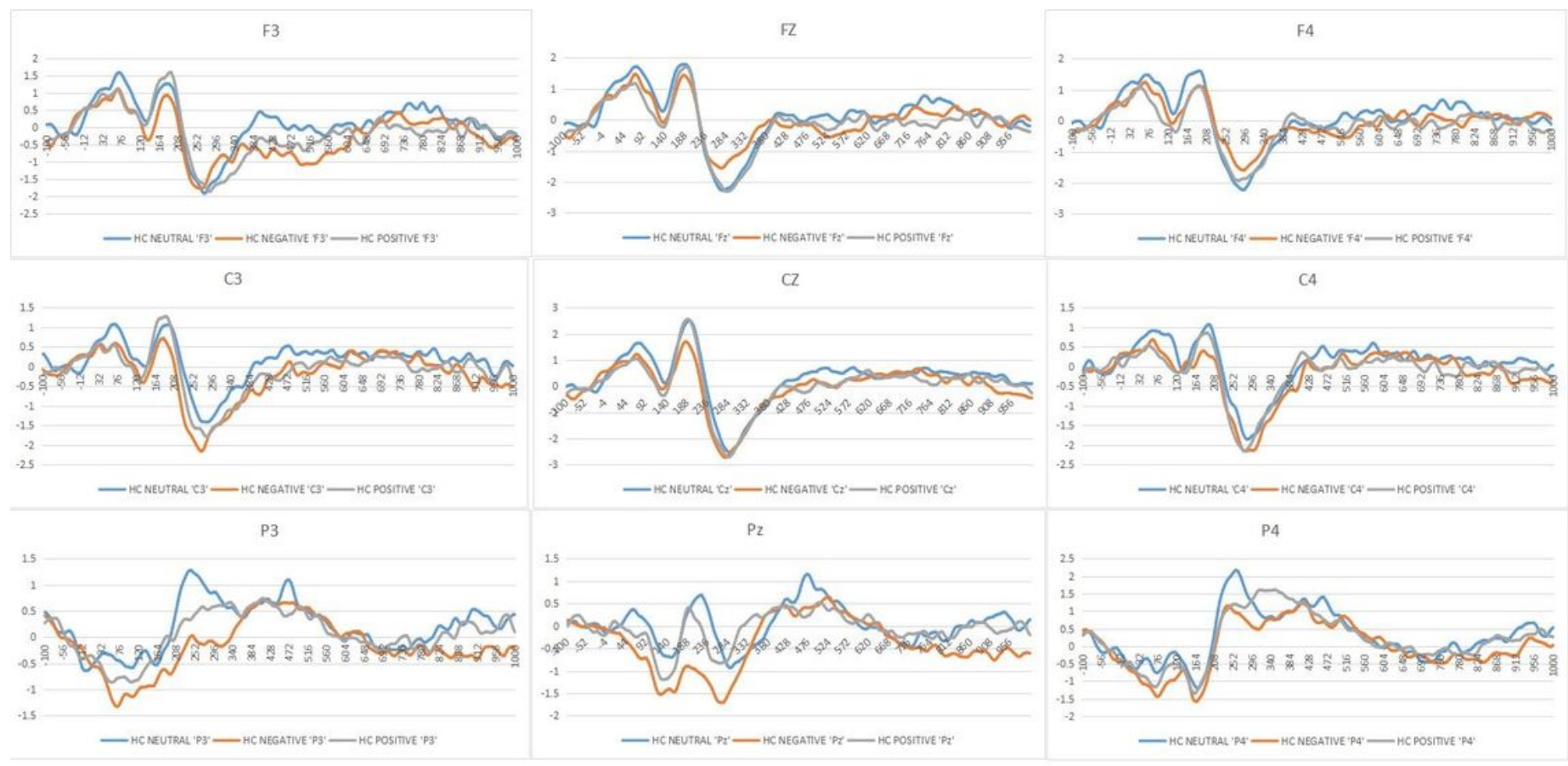

\section{Figure 1}

Grand mean ERPs in each affectine stimuli and neutral stimuli at the 9 ERP electrodes with time window of P200 for healthy controls (HCs or Healthy controls, Neutral or neutral stimuli, Negative or negative stimuli, Positive or positive stimuli). 


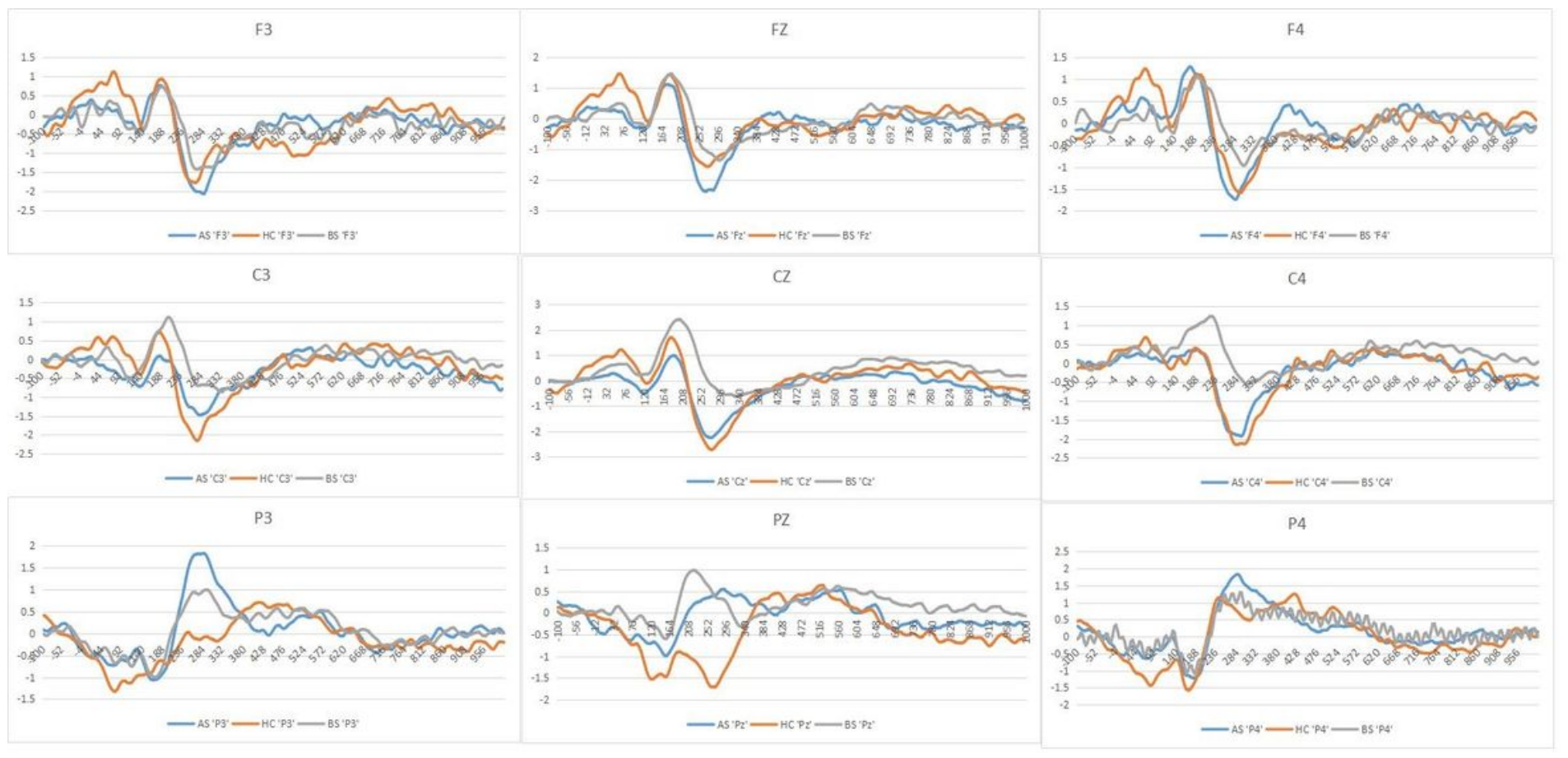

Figure 2

Grand averages in response to negative stimuli of the three groups at each of the 9 ERP locations employed in the present experiment stimuli (AS or postoperative group, BS or preoperative group, HCs or Healthy controls).
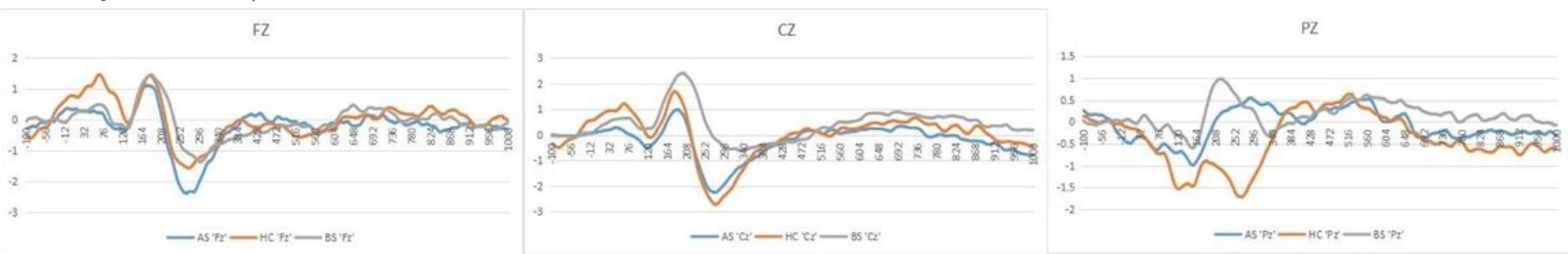

Negative stimuli
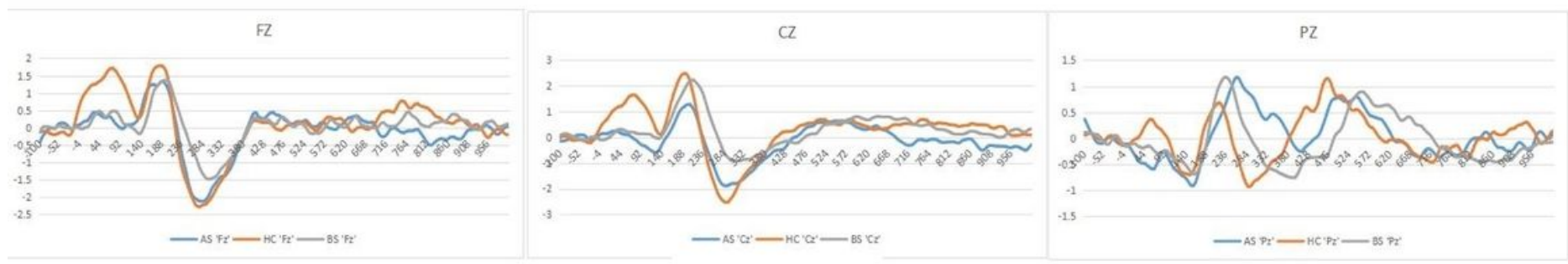

Neutral stimuli
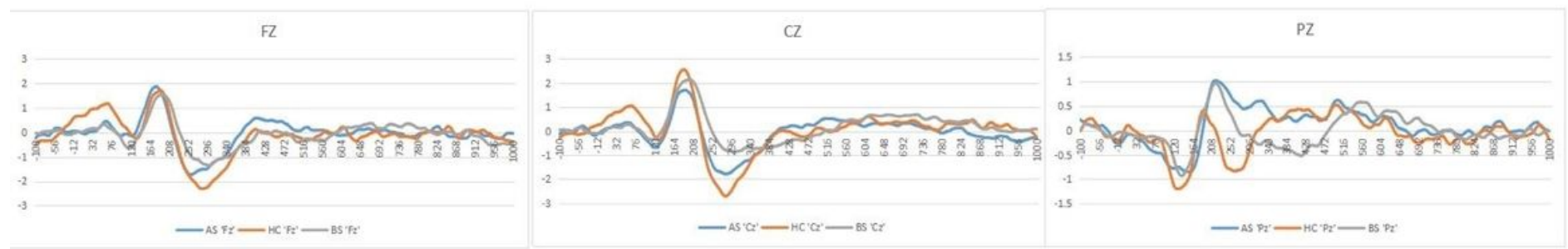

Positive stimuli 


\section{Figure 3}

Grand mean ERPs in each emotional stimuli and neutral stimuli at the three electrodes $(\mathrm{C} 3, \mathrm{Cz}, \mathrm{C} 4)$ with time window of P200 for three groups (AS or postoperative group, BS or preoperative group, HCs or Healthy controls). 